\title{
Enhanced muscle activity during lumbar extension exercise with pelvic stabilization
}

\author{
Ho-Seong Lee* \\ Department of Kinesiology and Medical Science, Graduate School, Dankook University, Cheonan, Korea
}

The purpose of this study was to investigate whether pelvic stabilization affects multifidus (MF) and iliocostalis lumborum (IL) muscle activities during dynamic extension exercise. Nine males (age, $25.1 \pm 6.3 \mathrm{yr} ;$ height, $176.6 \pm 2.4 \mathrm{~cm}$; body mass, $74.9 \pm 6.7 \mathrm{~kg}$ ) performed an isometric lumbar extension strength test and dynamic exercise in an upright seated position with or without pelvic stabilization. The electromyography and muscle strength of the MF and IL muscles were measured when the subjects performed the isometric lumbar extension strength test at the trunk angle $110^{\circ}, 146^{\circ}$, and $182^{\circ}$. In addition, the trunk extensor muscle activities were measured using $50 \%$ muscle strength of maximum isometric strength during a dynamic trunk extension exercise. The MF and IL muscle activities were significantly higher at $110^{\circ}, 146^{\circ}$, and $182^{\circ}$ with pelvic stabilization than that without pelvic stabilization during the isometric lumbar extension strength test $(P<0.05)$ and the dynamic exercise $(P<0.05)$. These results suggest that the lumbar extension exercise with pelvic stabilization may be more effective for MF and IL muscle activity compared to that without pelvic stabilization.

Keywords: Lumbar extension exercise, Muscle activity, Muscle strength dynamometer, Pelvic stabilization

\section{INTRODUCTION}

Low back pain (LBP) is one of the most common musculoskeletal disorders (van Tulder et al., 2000). In LBP patients, poor back muscle strength could be attributed to a higher proportion of type II fatigable fibers and/or atrophy of lumbar muscles (da Silva et al., 2009; Mannion et al., 2000). Resistance exercise training increases strength and endurance of back muscles (Mannion et al., 2001) and decreases pain and disability (Vincent et al., 2014) among patients with LBP.

Resistance training using lumbar extension machines has been commonly used to increase lumbar muscle strength and endurance to reduce LBP symptoms and disability (Fisher et al., 2013). Especially, lumbar extension exercise machines with pelvic stabilization mechanism are widely used for prevention and treatment of LBP. Lumbar extension machines with pelvic stabilization are composed of a leg restraint pad and a thigh-pelvic restraint pad to restrict the movement of the pelvis and hip extensor (San Juan et al., 2005).
Lumbar extensors are mainly composed of the erector spinae and multifidus muscle groups, and work cooperatively with gluteal and hamstring muscles during trunk extension (Graves et al., 1994). This compound movement is called lumbopelvic rhythm. From a position of full trunk flexion, the lumbar extensors (erector spinae and multifidus) and hip extensors (gluteals and hamstrings) work together to actively rotate the trunk through approximately $180^{\circ}$ in the sagittal plane (Cailliet, 1996). Lumbar movement accounts for approximately $72^{\circ}$ of this motion, whereas hip and pelvis movement accounts for the remaining $108^{\circ}$ (Pollock et al., 1989). In this respect, it seems that lumbar muscles requires stabilization of the pelvis to isolate the lumber extensors and minimize the contribution of the hip and leg muscles to effectively assess and train (Graves et al., 1992; Pollock et al., 1989).

Pelvic stabilization during trunk extension exercise also restricts the involvement of the hip extensors and provides optimized recruitment of lumbar extensors (Graves et al., 1994; Smidt et al., 1983). Graves et al. (1994) reported that lumbar extension exercise
${ }^{*}$ Corresponding author: Ho-Seong Lee

Department of Kinesiology and Medical Science, Graduate School, Dankook

University, 119 Dandae-ro, Dongnam-gu, Cheonan 31116, Korea

Tel: +82-41-550-3838, Fax: +82 41-559-7915, E-mail: hoseh28@dankook.ac.kr

Received: November 12, 2015 / Accepted: December 10, 2015
This is an Open Access article distributed under the terms of the Creative Commons Attribution Non-Commercial License (http://creativecommons.org/licenses/by-nc/3.0/) which permits unrestricted non-commercial use, distribution, and reproduction in any medium, provided the original work is properly cited. 
training with pelvic stabilization on a lumbar dynamometer effectively developed lumbar extension strength, while training without the stabilization was ineffective. Choi et al. (2005) also reported that a 12-week postoperative lumbar extension training with pelvic stabilization to herniated disc patients improved multifidus (MF) and longissimus muscle mass, decreased pain, and higher rates of return to work compared without pelvic stabilization.

In contrast, several studies have reported that there were no differences in lumbar extensor muscle activity between lumbar extension exercise with pelvic stabilization and that without stabilization. Mayer et al. (2002) showed that lumbar extension exercise on a lumbar extension machine increased in strength of lumbar extensor, even in the absence of pelvic stabilization. Benson et al. (2002) also reported that there was no difference in lumbar extension muscle activity between the stabilized and unstabilized conditions by using the roman chair. Despite many studies have been performed on machines for lumbar extension exercise with or without pelvic stabilization, there is still controversy as to whether pelvic stabilization affects lumbar extensor muscle activity.

Therefore, the purpose of this study was to investigate whether pelvic stabilization affected the MF and iliocostalis lumborum (IL) muscle activities during isometric lumbar strength test and dynamic exercise. We hypothesized that pelvic stabilization would enhance the activities of MF and IL muscles during isometric lumbar strength test and dynamic exercise.

\section{MATERIALS AND METHODS}

\section{Design}

A within-subject, repeated measures design was used to examine surface electromyography (EMG) of MF and IL muscle with or without pelvic stabilization on during an isometric lumbar extension strength test and a dynamic exercise. Isometric lumbar extension strength test and dynamic exercise were performed in the seated upright position under two conditions of with or without pelvic stabilization using a lumbar extension machine. The same investigator performed the procedures and the tasks with all subjects to ensure uniformity.

\section{Participants}

Nine males (age, 25.1 $\pm 6.3 \mathrm{yr}$; height, $176.6 \pm 2.4 \mathrm{~cm}$; body mass, $74.9 \pm 6.7 \mathrm{~kg}$ ) were recruited from a university setting and participated in this study. Potential subjects were excluded from the project if they fit any of the following criteria (San Juan et al., 2005): (a) under eighteen or over fifty years of age; (b) history of chronic LBP; (c) currently experiencing LBP; (d) history of lumbar spine pathologies; (e) knee or hip conditions contraindicating the use of pelvic restraint; (f) history of cardiovascular disease without consent from a physician; and $(\mathrm{g})$ orthopedic or cardiovascular contraindications to resistance training. This study was approved by the Ethics Committee of Dankook University, in accordance with the ethical standards of the Declaration of Helsinki (DKU 2015-05-011). Participants provided written informed consents.

\section{Procedure}

A week before the period, all participants then performed manually resisted isometric back extension trials to elicit the maximal voluntary contraction (MVC) level for the MF and IL (McGill, 1992). The MF was tested with the participant prone, arms crossed over the chest and attempting to perform back extension and rotation to the opposite side while the investigator resisted the motion. IL was tested with the participant supine, the pelvis laterally flexed in relation to the trunk and attempting to perform lateral flexion to the opposite side while the investigator resisted the motion. The maximum EMG signal of any of the three trials was designated as the MVC and used to normalize the subsequent EMG data.

Isometric lumbar extensor strength test and dynamic exercise sessions separated by one week were required. Isometric lumbar extension strength test and dynamic exercise were using a lumbar extension exercise system (LEX; Daeyang Mechanics, Seoul, Korea) between pelvic stabilized and unstabilized conditions. Isometric lumbar extension strength values were used to collect the EMG

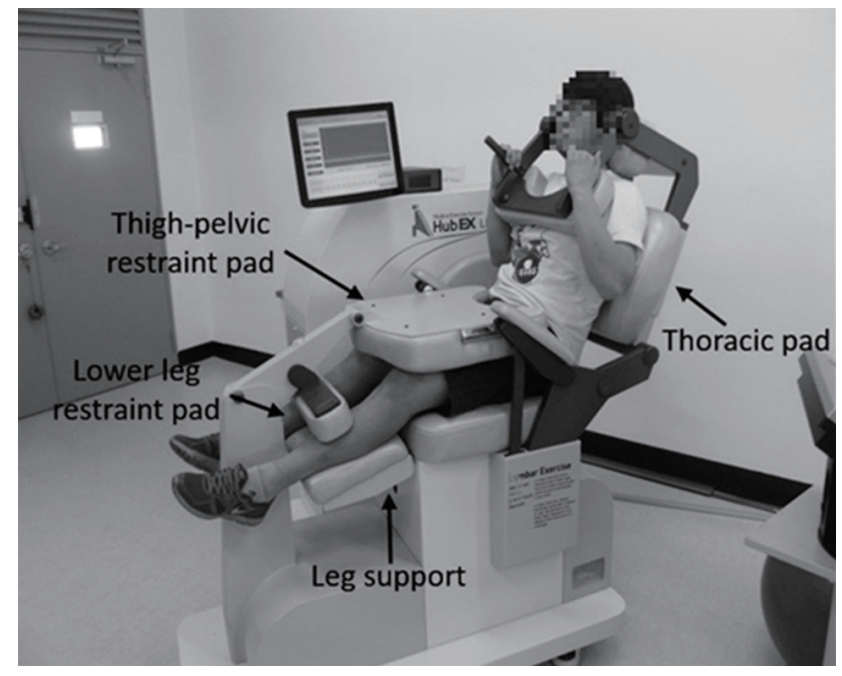

Fig. 1. Pelvic stabilization mechanisms on the lumbar extension exercise dynamometer. 
data and to determine the load for the dynamic exercise. Subjects were seated in the LEX in an upright position in order to isometric lumbar extension strength test with pelvic stabilized condition. The knees were positioned so that the thighs were parallel to the seat. The lower legs were placed in the leg support and fixed a lower leg restraint pad. The anterior portion of the thigh and pelvic were secured with the thigh-pelvic restraint pad over the anterior thigh and pelvis (Fig. 1). Next, passive lumbar ranges of motion in the sagittal plane and centers of torso mass were determined. Then, the subjects performed a series of submaximal isometric strength tests and light dynamic exercise for familiarization to the device. After a 15-min rest period, the subjects were positioned in the lumbar dynamometer as described above, maximum voluntary isometric lumbar extension torque output was measured at three positions at $110^{\circ}, 146^{\circ}$, and $182^{\circ}$ of lumbar extension. When ready to test, the subject was requested to build up to maximal tension over 2-3 sec and to maintain the contraction for a further $1 \mathrm{sec}$ with rest interval $10 \mathrm{sec}$ between each angle. During the test, the subjects were verbally encouraged to generate maximum torque (Graves et al., 1994). Isometric lumbar extension strength test in the unstabilized pelvic position was performed in similar condition to that the stabilized pelvic position. However, movement of the pelvis was restricted that the thigh-pelvic restraint pad, leg support and leg restraint pad were not used.

After the isometric strength test, subjects rested in a lying position for 15-20 min, and then performed dynamic exercise. Dynamic exercise conducted under the same condition as in the isometric lumbar strength test. Dynamic exercise was performed from $110^{\circ}$ to $182^{\circ}$ of extension and load set at $50 \%$ of their individual maximum isometric torque. The subjects were instructed to perform 20 repetitions and spend 2 sec completing each eccentric and concentric phase. Dynamic exercise in the unstabilized pelvic position was performed in similar condition to that the stabilized pelvic position. The order for the two exercise conditions was balanced across subjects.

\section{EMG assessment}

The EMG signal was collected using Noraxon Telemyo 2000 System (Noraxon USA Inc., Scottsdale, AZ, USA), including receiver, transmitter, and USB A/D converter at a sampling rate of $1,000 \mathrm{~Hz}$. Common mode ratio was $>100 \mathrm{~dB}$, baseline noise was $<1 \mathrm{mV}$, and input impedance was $>100 \mathrm{mOhms}$. The raw data were full-wave rectified, smoothed at $50 \mathrm{~ms}$ RMS algorithm, filtered, and normalized as a percentage of the middle 3 sec of the MVC data using the myoresearch software XP (Noraxon USA
Inc.).

Surface EMG signals were recorded from the right lumbar MF and IL muscles. Electrodes for the MF were attached right side of the $\mathrm{L} 3-\mathrm{L} 4$ spinous process, approximately $3 \mathrm{~cm}$ from the midline of the torso. The IL electrodes were placed parallel to the line between the posterior superior iliac spine and the lateral border of the muscle at the 12th rib ( $\mathrm{Ng}$ et al., 1997). Before application of the surface electrodes, the subjects' skin was shaved if necessary, and wiped with alcohol to reduce skin impedance (Marshall and Murphy, 2005). The centre-to-centre electrode distance was 2.5 $\mathrm{cm}$. Electrodes were positioned above the midpoint of the muscle belly, parallel to the direction of the muscle fibers. They were further secured to the skin with adhesive tape together with the preamplifier to reduce motion artefacts (Karamanidis et al., 2004).

\section{Statistical analysis}

Measured data, we used the paired $t$-test for comparisons of MF and IL muscle activities in restraint conditions (stabilized pelvis and unstabilized pelvis) during isometric lumbar extension strength test and dynamic exercise. No statistical comparisons were made between the two muscles. IBM SPSS ver. 18.0 (IBM Co., Armonk, NY, USA) was used for the statistical analysis with a significance level of $\alpha=0.05$.

\section{RESULTS}

Changes in the muscle activity of $\mathrm{MF}$ at $110^{\circ}, 146^{\circ}$, and $182^{\circ}$ with and without pelvic stabilization are shown in Fig. 2. The muscle activity of the MF was significantly higher at $110^{\circ}, 146^{\circ}$, and $182^{\circ}$ in pelvic stabilized condition than pelvic unstabilized

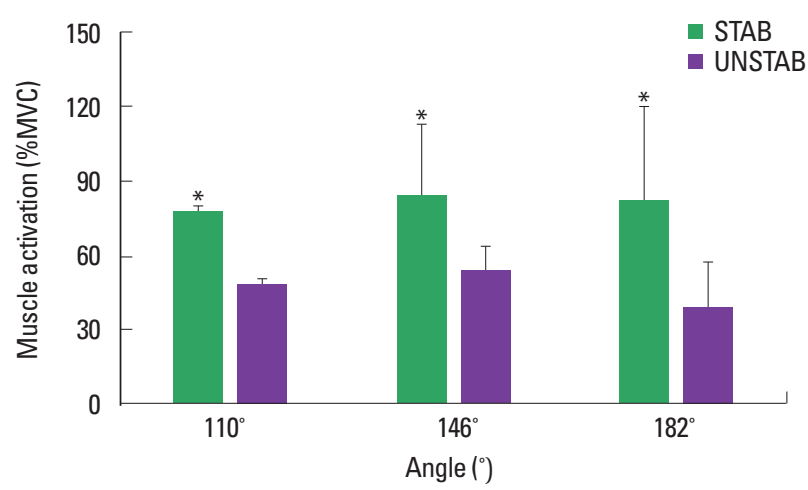

Fig. 2. Comparison of the multifidus muscle activity at $110^{\circ}, 146^{\circ}$, and $182^{\circ}$ during isometric lumbar extension strength testing with the pelvis stabilized (STAB) and unstabilized (UNSTAB) conditions. Values are presented as mean \pm standard deviation. ${ }^{*} P<0.05$ vs. UNSTAB. MVC, maximal voluntary contraction. 


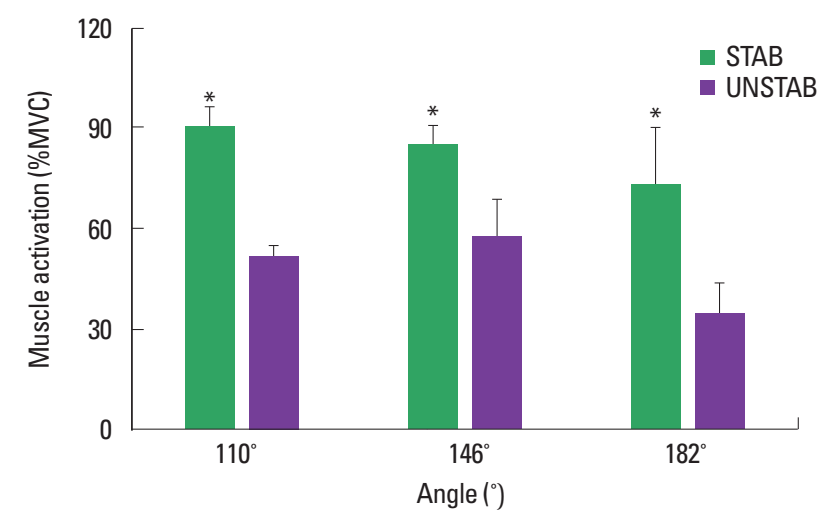

Fig. 3. Comparison of the iliocostalis lumborum muscle activity at $110^{\circ}, 146^{\circ}$, and $182^{\circ}$ during isometric lumbar extension strength testing with the pelvis stabilized (STAB) and unstabilized (UNSTAB) conditions. Values are presented as mean \pm standard deviation. ${ }^{*} P<0.05$ vs. UNSTAB. MVC, maximal voluntary contraction.

condition during the isometric lumbar extension strength test $(P<0.05)$. Changes in the muscle activity of IL at $110^{\circ}, 146^{\circ}$, and $182^{\circ}$ with and without pelvic stabilization are shown in Fig. 3 . The muscle activity of the IL was significantly higher at $110^{\circ}$, $146^{\circ}$, and $182^{\circ}$ in pelvic stabilized condition than pelvic unstabilized condition during the isometric lumbar extension strength test $(P<0.05)$. Changes in the muscle activities of MF and IL with and without pelvic stabilization during dynamic exercise are shown in Fig. 4. The muscle activities of the MF and IL were significantly higher at pelvic stabilized condition than pelvic unstabilized condition during the dynamic exercise $(P<0.05)$.

\section{DISCUSSION}

The major finding of the present study was that MF and IL muscle activities were higher in isometric lumbar strength test and dynamic exercise with pelvic stabilization than that without pelvic stabilization. This result supports our hypothesis that pelvic stabilization enhances activity of MF and IL muscles during isometric lumbar strength test and dynamic exercise.

In contrast, Udermann et al. (1999) reported that the EMG activity of the lumbar extensors were not different between pelvic stabilized and unstabilized conditions. These differences may be related to difference in pelvic stabilization mechanisms among lumbar extension machines. According to Pollock et al. (1989), isolation of the lumbar extensors through restraining both the legs and pelvis was shown to reduce pelvic movement compared with less strict restraints. This process helps eliminate the contribution of the hip extensors during trunk extension (Graves et al.,

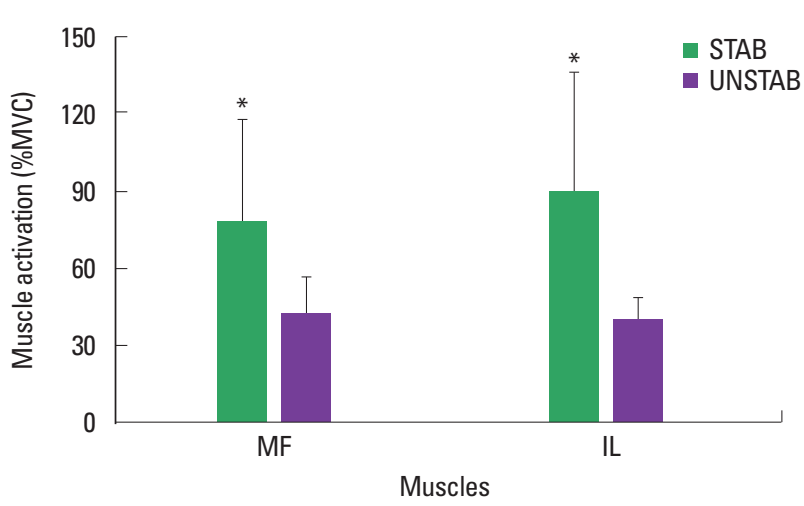

Fig. 4. Comparison of the lumbar multifidus (MF) and iliocostalis lumborum (IL) during dynamic exercise with the pelvis stabilized (STAB) and unstabilized (UNSTAB) conditions. Values are presented as mean \pm standard deviation. ${ }^{*} P<0.05$ vs. UNSTAB. MVC, maximal voluntary contraction.

1994). Therefore, the results of the present study were due to provision for a completely fixed both the legs and pelvis.

Lumbar muscles are dedicated to intersegmental stability and generating movement so that weakness of lumbar muscles leads to LBP, functional disability, instability to reduce mobility (Dolan and Adams, 1993). The goal of exercise in patients with LBP is to improve back extension strength and mobility, alleviate functional disability (Risch et al., 1993). According to a previous study, lumbar extension training with pelvic stabilization increased back muscle strength and decreased pain and disability in patients with LBP (Süüden et al., 2008). These findings are in accordance with the results of a study conducted by Graves et al. (1994), which found lumbar extension exercise with pelvic stabilization is more effective method for increasing lumbar extension strength than that without pelvic stabilization. Another study also reported that pelvic stabilization significantly increased activities of the trunk extensors during isometric trunk muscle exercise (Shirado et al., 1995). In the present study, muscle activities of MF and IL were significantly higher in pelvic stabilized condition than that in pelvic unstabilized condition. Our finding clearly indicates that pelvic stabilization is essential to do the lumbar muscles effectively, and that such stabilization may increase lumbar extension strength, effectively.

Pollock et al. (1989) and Graves et al. (1994) have speculated that the relatively large increases in the ability of the isolated lumbar extensor muscles to generate torque, particularly in the more extended portions of the range-of-motion because these muscles are initially very weak. The lumbar extensors are rarely recruited during normal daily activities so that they seldom encounter an 
overload stimulus required to gain strength. If pelvic stabilization is essential to strengthen lumbar extensors, and so even those who perform regular weight training may suffer from disuse atrophy of the lumbar muscles, and should include lumbar extension training in their workout regimes to keep low back health (Koumantakis et al., 2005). This assertion is supported by a recent case study (Süïden et al., 2008), showing that a participant who had lost an average of $42 \%$ of his lumbar strength during $10 \mathrm{yr}$, despite regularly performing heavy deadlifts, squats, bent-over rows, and other weight training exercises that load the lumbar spine.

The limitations of the present study should be taken into consideration. Our results cannot necessarily be generalized to LBP patients who have different patterns of lumbar muscle activity. Further investigation is necessary to determine whether pelvic stabilization increases muscle activity in patients with LBP during lumbar extension exercise to reduce LBP symptoms.

In conclusion, our study demonstrated that the isometric lumbar extension strength test and dynamic exercise with the pelvic stabilized condition were shown to be more effective exercise for MF and IL muscle activity, compared to pelvic unstabilized condition. The pelvic stabilized condition would be more effective in training lumbar extension muscles through enhanced muscle activity. This information could be used to provide back muscle strength training, prevention and rehabilitation of LBP.

\section{CONFLICT OF INTEREST}

No potential conflict of interest relevant to this article was reported.

\section{ACKNOWLEDGMENTS}

This study was performed in the Institute of Medi-Sports at the Dankook University (BK21 Plus), Cheonan, Korea.

\section{REFERENCES}

Benson ME, Smith DR, Bybee RF. The muscle activation of the erector spinae during hyperextension with and without the pelvis restrained. Phys Ther Sport 2002;3:165-174.

Cailliet R. Soft tissue pain and disability. 3rd ed. Philadelphia: F. A. Davis Company; 1996.

Choi G, Raiturker PP, Kim MJ, Chung DJ, Chae YS, Lee SH. The effect of early isolated lumbar extension exercise program for patients with herniated disc undergoing lumbar discectomy. Neurosurgery 2005;57:764-
772.

da Silva RA, Larivière $C$, Arsenault AB, Nadeau S, Plamondon A. Pelvic stabilization and semisitting position increase the specificity of back exercises. Med Sci Sports Exerc 2009;41:435-443.

Dolan P, Adams MA. Influence of lumbar and hip mobility on the bending stresses acting on the lumbar spine. Clin Biomech (Bristol, Avon) 1993;8:185-192.

Fisher J, Bruce-Low S, Smith D. A randomized trial to consider the effect of Romanian deadlift exercise on the development of lumbar extension strength. Phys Ther Sport 2013;14:139-145.

Graves JE, Fix CK, Pollock ML, Leggett SH, Foster DN, Carpenter DM. Comparison of two restraint systems for pelvic stabilization during isometric lumbar extension strength testing. J Orthop Sports Phys Ther 1992;15:37-42.

Graves JE, Webb DC, Pollock ML, Matkozich J, Leggett SH, Carpenter DM, Foster DN, Cirulli J. Pelvic stabilization during resistance training: its effect on the development of lumbar extension strength. Arch Phys Med Rehabil 1994;75:210-215.

Karamanidis K, Arampatzis A, Brüggemann GP. Reproducibility of electromyography and ground reaction force during various running techniques. Gait Posture 2004;19:115-123.

Koumantakis GA, Watson PJ, Oldham JA. Trunk muscle stabilization training plus general exercise versus general exercise only: randomized controlled trial of patients with recurrent low back pain. Phys Ther 2005;85:209-225.

Mannion AF, Käser L, Weber E, Rhyner A, Dvorak J, Müntener M. Influence of age and duration of symptoms on fibre type distribution and size of the back muscles in chronic low back pain patients. Eur Spine J 2000;9:273-281.

Mannion AF, Taimela S, Müntener M, Dvorak J. Active therapy for chronic low back pain part 1. Effects on back muscle activation, fatigability, and strength. Spine (Phila Pa 1976) 2001;26:897-908.

Marshall PW, Murphy BA. Core stability exercises on and off a Swiss ball. Arch Phys Med Rehabil 2005;86:242-249.

Mayer JM, Verna JL, Manini TM, Mooney V, Graves JE. Electromyographic activity of the trunk extensor muscles: effect of varying hip position and lumbar posture during Roman chair exercise. Arch Phys Med Rehabil 2002;83:1543-1546.

McGill SM. A myoelectrically based dynamic three-dimensional model to predict loads on lumbar spine tissues during lateral bending. J Biomech 1992;25:395-414.

$\mathrm{Ng}$ JK, Richardson CA, Jull GA. Electromyographic amplitude and frequency changes in the iliocostalis lumborum and multifidus muscles during a trunk holding test. Phys Ther 1997;77:954-961.

Pollock ML, Leggett SH, Graves JE, Jones A, Fulton M, Cirulli J. Effect of 
resistance training on lumbar extension strength. Am J Sports Med 1989;17:624-629.

Risch SV, Norvell NK, Pollock ML, Risch ED, Langer H, Fulton M, Graves JE, Leggett SH. Lumbar strengthening in chronic low back pain patients. Physiologic and psychological benefits. Spine (Phila Pa 1976) 1993;18:232-238.

San Juan JG, Yaggie JA, Levy SS, Mooney V, Udermann BE, Mayer JM. Effects of pelvic stabilization on lumbar muscle activity during dynamic exercise. J Strength Cond Res 2005;19:903-907.

Shirado O, Ito T, Kaneda K, Strax TE. Electromyographic analysis of four techniques for isometric trunk muscle exercises. Arch Phys Med Rehabil 1995;76:225-229.

Smidt G, Herring T, Amundsen L, Rogers M, Russell A, Lehmann T. Assessment of abdominal and back extensor function. A quantitative approach and results for chronic low-back patients. Spine (Phila Pa 1976) 1983;8:211-219.
Süüden E, Ereline J, Gapeyeva H, Pääsuke M. Low back muscle fatigue during Sørensen endurance test in patients with chronic low back pain: relationship between electromyographic spectral compression and anthropometric characteristics. Electromyogr Clin Neurophysiol 2008;48:185-192.

Udermann BE, Graves JE, Donelson RG, Ploutz-Snyder L, Boucher JP, Iriso JH. Pelvic restraint effect on lumbar gluteal and hamstring muscle electromyographic activation. Arch Phys Med Rehabil 1999;80:428431.

van Tulder M, Malmivaara A, Esmail R, Koes B. Exercise therapy for low back pain: a systematic review within the framework of the cochrane collaboration back review group. Spine (Phila Pa 1976) 2000;25:27842796.

Vincent HK, George SZ, Seay AN, Vincent KR, Hurley RW. Resistance exercise, disability, and pain catastrophizing in obese adults with back pain. Med Sci Sports Exerc 2014;46:1693-1701. 